\title{
Applications incorporating occupant behavior into building simulation
}

\author{
Clinton J. Andrews' $(\varangle)$, Bing Dong ${ }^{2}$ \\ (Guest Editors) \\ 1. Edward J. Bloustein School of Planning and Public Policy, Rutgers University, 33 Livingston Ave, New Brunswick, NJ 08901, USA \\ 2. Department of Mechanical Engineering, The University of Texas, San Antonio, One UTSA Circle, San Antonio, TX 78023, USA
}

๑) Tsinghua University Press and Springer-Verlag GmbH Germany 2017

The building simulation community is paying increased attention to occupant behavior. Many interdependencies between building systems and their human users exist. Behavioral factors often affect building performance, even as building systems affect the comfort, satisfaction, and productivity of occupants. Researchers have proposed variety of strategies for incorporating consideration of occupant behavior into building applications such as performance modeling and simulation, equipment controls and whole building energy optimization. It is also timely to explore the fit between modeling approaches and specific applications.

The guest editors of this topical issue met at meetings of the International Energy Agency's Annex 66 expert working group on definition and simulation of occupant behavior in buildings (www.www.annex66.org). There, we encountered much interesting and creative research on occupant behavior modeling, but we were not sure whether it was relevant to practice, and whether there were viable occupant driven applications. Thus this topical issue was born. We sought papers on applications of building simulation to building design, controls, operations, retrofits, equipment design, and public policy. By the time the dust cleared in the peer review process, we had 17 strong papers that covered most of these application areas.

Building types featured in this collection include offices (5 papers), multifamily residences (4), single-family residences (2), dormitories (1), museums (1), and even a cruise ship (1). One paper focuses on the urban scale. One paper focuses on water consumption and the rest examine energy consumption, often in combination with thermal and lighting comfort. Particularly interesting are two papers examining ultra-low energy buildings, where efficient building systems magnify the relative importance of occupant behaviors in determining energy performance.

Different stages in the building life cycle, from design, to operation, to retrofit, have distinct occupant behavior simulation-modeling requirements. Several papers offer insights about how to make building designs more behaviorally robust, meaning that the design anticipates the likely variability and patterns of occupant behavior. Others focus on important operational concerns including wayfinding for emergency evacuation, optimal scheduling of public spaces, enhanced model predictive control of building systems, smart building responses to dynamic pricing on a smart electric power grid, and occupant sensitivity to electric load shedding. A third group considers how occupant behavior changes the relative attractiveness of alternative energy efficiency retrofit strategies.

Three papers present methodological advances that make dynamic simulations of building-occupant systems more tractable. These represent important milestones in bringing applied modeling practices into closer proximity with advanced modeling research.

Overall, this collection of papers shows that occupant behavior modeling has many valuable and important applications. The range of purposes and contexts illustrated here confirm that occupant behavior is, or should be, a core consideration in most building simulation modeling efforts. It is no longer just a research topic-it is becoming an element of practice. Much work is still needed to make this type of modeling cost-effective and user-friendly. The tasks include creating robust and transferable data sets on contextualized occupant behavior, identifying modeling approaches that are "just good enough" for the specific application and fit-for-purpose, and improving the interoperability of occupant-behavior and building-simulation models.

E-mail: CJA1@RUTGERS.EDU 